\title{
Sustainable Highway Operations: High Occupancy Lane and Movable Lane
}

\author{
Jooyoung Kim ${ }^{1}$, Sangung Park ${ }^{2}$, Sungyong $\mathrm{Na}^{3}$ and Seungjae Lee \\ 1,2,3,4 Department of Transportation Engineering \\ University of Seoul, (90 Jeonnong-dong, Dongdaemun-gu, Seoul, Korea \\ TEL: (+82)-2-6490-5661 \\ ${ }^{1}$ trafficplan@naver.com, ${ }^{2}$ hazsang@naver.com, ${ }^{3}$ traffic_na@naver.com, \\ ${ }^{4}$ sjlee@uos.ac.kr
}

\begin{abstract}
Two managed lane schemes, the high occupancy vehicle lane (HOV) and the movable lane operations, were analyzed to determine their suitability as a sustainable highway operation. VISSIM, a microscopic traffic simulation program, was used for this study, and parameters representing driver behavior in the car following model were calibrated according to a macroscopic speed and flow relationship based on measured data. The analysis results showed that bus travel speed was improved in the HOV operation, while movable lane operation also considerably improved car travel speed.
\end{abstract}

Keywords: sustainable highway operation, managed lane scheme, HOV lane, Movable lane, VISSIM, calibration

\section{Introduction}

\subsection{Background and Aim}

Sustainable development encompasses balanced growth in economic, sociable and environmental ways within the limits of marginal capacities for future generations while fulfilling the needs of the current generation (1).

The United Nations Framework Convention on Climate Change, which was held in Bali, Indonesia, in December 2007, is a comprehensive plan of action to be taken globally, nationally and locally in every area in which humans impact on the environment. Transportation cannot be excluded. Sustainable transportation mainly aims to reduce energy consumption and greenhouse gases while increasing traffic safety. The policies for sustainable transportation include reducing motor car trips and promoting public transport and green transport (2).

However, transport conditions in many large cities such as Seoul remain far removed from the goal of the sustainable transportation policies. Over the past forty years, the number of car owners has dramatically increased as income levels climbed. The number of cars in the nation nearly doubled from 8,469,000 in 1995 to $15,850,000$ in 2006. According to KOSTA (2007), the daily average travel distance of cars rose slightly from $44.2 \mathrm{~km}$ in 2000 to $45.9 \mathrm{~km}$ in 2006 . When it comes to transportation energy consumption, the annual average figure increased by $6.3 \%$ between 1990 and 2006. In addition, the transportation energy consumption forms $21 \%$ of the national energy consumption. Within the transportation fields, highways consume the greatest energy at $78 \%$ and railways the smallest at $1 \%$. (3).

Therefore, this paper proposes and evaluates alternatives in both public transport and motor vehicles in order to reduce the energy consumption and greenhouse gases in 
automotive vehicles that are the main area of sustainable transportation in our country. Firstly, as an alternative plan to increase the usage of public transport, the high occupancy vehicle (HOV) lane is evaluated. Secondly, to enhance the energy efficiency of cars, the effect of adopting a movable lane as a managed lane is analyzed. In addition, this paper introduces a method to calibrate realistic driver behaviors by fitting parameters in the microscopic car following model into a macroscopic flow-speed relationship based on counted data.

\subsection{Scope}

This study examined the area of the Gyeongbu (Seoul-Busan) Expressway since the HOV lane between Hannam Bridge (Seoul Toll Gate) and Osan Interchange of the Gyeongbu Expressway has recently been operating during weekdays.

\section{Simulation Model Construction}

\subsection{Review of Simulation Models}

There are two models to analyze traffic flows, macroscopic flow models and microscopic flow models, although a third model type, mesoscopic flow models, can be included between them. Macroscopic flow models analyze individual attributes as a group and concentrate on traffic conditions systemically. The relational equations of traffic volume-density-speed are mainly used for algorithms in these models. On the other hand, microscopic flow models analyze traffic conditions individually in comparatively smaller areas. Car follow models and gap acceptance models are used in these models.

In this paper, microscopic models are used in order to represent realistic conditions in two regimes of highway sections, bus-only lanes and mixed traffic lanes, in order to evaluate a managed lane scheme. CORSIM, VISSIM and PARAMICS could be used for this study but we compare the features in each of the models, and then select one for this study.

\subsection{General Review}

CORSIM is Developed by the Federal Highway Administration, (Ed- the abbreviation is unnecessary as it is not used anywhere else in the paper) CORSIM is one of the most commonly used traffic operations programs. PARAMICS is Developed by Quadstone Limited in Scotland, PARAMICS is a traffic operation program used to analyze the movement and behavior of individual vehicles and transit on local arterial and regional freeway networks. VISSIM is Developed by Planung Transport Verkehr (PTV) in Germany, VISSIM is one of the most sophisticated microscopic simulation programs. It is used to analyze driver behavior and multi-modal transit operations, planning, and forecasting, as well as providing a 3-D simulation.

\subsection{Comparison}

The times spent on inputting initial data are similar in these three programs. PARAMIC and VISSIM have more input parameters so that they can improve the accuracy of results. Furthermore, CORSIM is limited in terms of the refinements for representing interchange, which puts it at a disadvantage in comparison to PARAMIC and VISSIM. The program manuals for these simulation models do not satisfactorily explain the adjustable parameters in various traffic conditions. Therefore, the changes made to the parameters depend on engineering judgments as well as the measured data. In terms of the level of service analysis at an intersection, unlike CORSIM, which provides control delay files and turn movement as output, PARAMICS only provides the total delay. 
However, VISSIM provides total delay, turn movement and also each link's delay. (4) In terms of the level of service analysis in a freeway, CORSIM does not provide the density per each lane, although the other two programs do. Overall, CORSIM needs a shorter setup time than the other two models. However, it suffers the disadvantages of inaccuracies on lamp metering and backups in high volume traffic areas. (5) While both CORSIM and PARAMICS use link-based routing, VISSIM uses path-based routing, which makes its results more reliable and accurate for intersection analysis. In the aspect of accuracy and detail of the output data generated by the input data, VISSIM is the superior model and, therefore, was used to evaluate the managed lane schemes in this paper.

\subsection{Introduction of VISSIM}

VISSIM (ED- you've already described this) is a microscopic, multi-purpose, traffic flow simulation program for driver behavior and network analysis based on a car following model. In terms of general characteristics, realistic road structures such as roadway geometrics, intersection configurations and traffic signal control can be represented. Driver behaviors that are similar to those in real situations can be described as well. Specifically, vehicles using defaulted lanes such as exclusive bus lanes, among others, can be represented and public transport attributes such as bus stop time, boarding time, among others, can be defined by users. However, VISSIM is affected by geometric design and signal as the traffic flow is microscopic. Therefore, not only accurate network coding but also numerous calibration steps are needed to ensure model reliability. As mentioned before, the parameters that can be modified for many different traffic conditions are adjusted by the user's judgments in the present because little information is provided about the default parameters in the user manual.

\subsection{Model Calibration}

In this paper, VISSIM was selected to evaluate the managed lane schemes. Calibration was carried out by adjusting the parameters of the car following model that analyzes driver behavior based on the flow-speed relationship. The process of calibration is shown in Figure 1. The basic data was inputted for the study area from the Seoul Toll Gate to Osan Interchange of the Gyeongbu Expressway, as shown in Figure 2. The geometrics data, including the length $(6.4 \mathrm{~km})$ and the number of lanes (4 lanes in each direction), were input followed by the width of the lanes, vertical slope, links, and connectors.

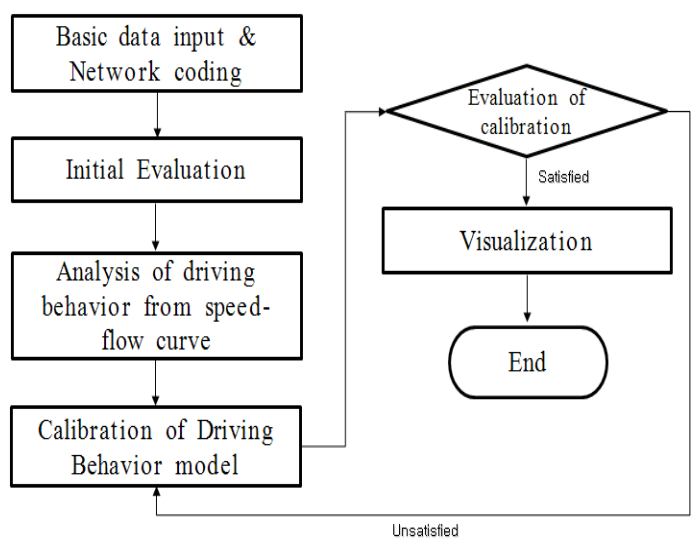

Figure 1. Calibration Procedure

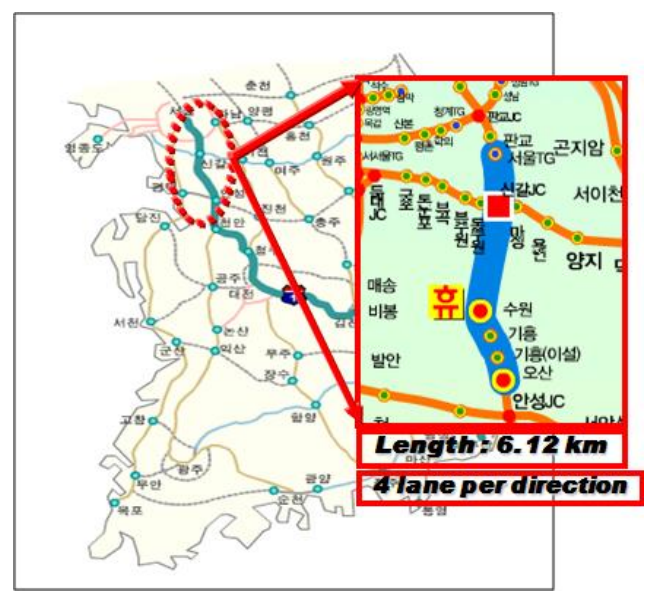

Figure 2. Study Area

In the initial evaluation, which measured the flow-speed data and default parameters defined by VISSIM program, a significant gap was found between the field data and the 
simulation results in terms of frequency and travel speed, as shown in Figure 3.

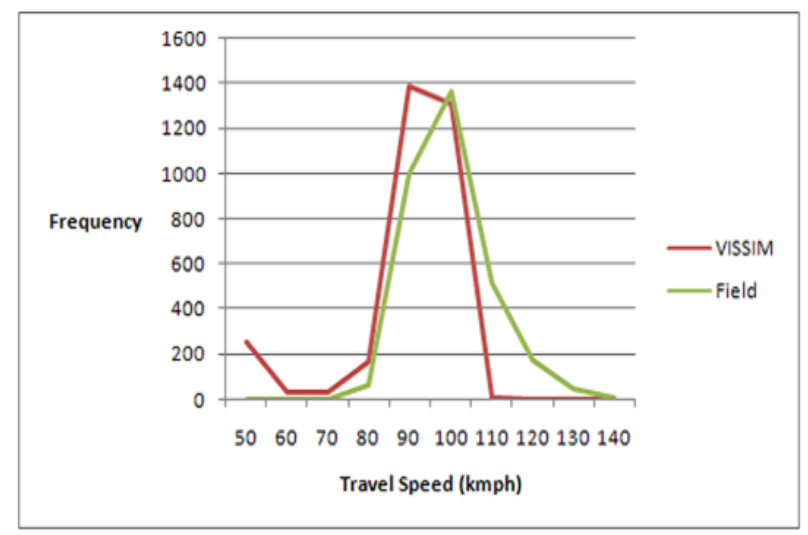

Figure 3. Initial Evaluation

This might be because the default parameters defined by VISSIM program represent driver behavior and traffic flow in Germany, where the program was developed. In order to understand how parameters are defined, the flow-speed relationship must be analyzed. Therefore, flow-speed relationships in Germany and South Korea are analyzed in this paper. The flow-speed relationship in the German Freeway is shown in Figure 4. The highest speed is $140 \mathrm{~km} / \mathrm{h}$ and the highest volume per hour per lane is 1,800.(6) The flowspeed relationship in the Gyeongbu Expressway, South Korea, is shown in Figure 5. The highest speed is $120 \mathrm{~km} / \mathrm{h}$ and the highest volume per hour per lane is 2,200 . The comparison of the flow-speed relationship in the two countries revealed that the highest volume per hour per lane in Germany was 400 less than that in South Korea, while the highest speed was $20 \mathrm{~km} / \mathrm{h}$ faster in Germany. These facts demonstrated the greater aggressiveness of the German driving behavior, compared to the Korean one.

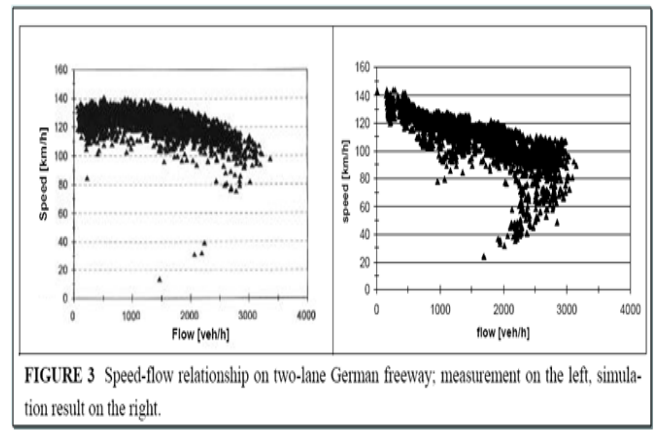

Figure 4. Flow-Speed Relationship in Germany(6)

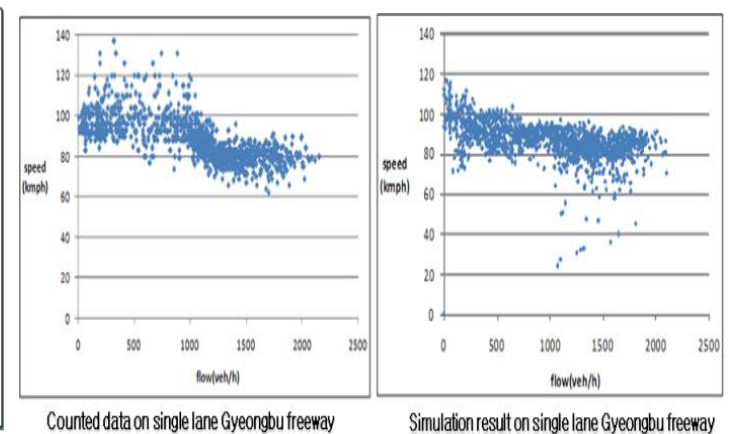

Figure 5. Flow-Speed Relationship in South Korea

\subsection{Parameter Adjustment}

The car following model of VISSIM is based on behavior-based parameters, as shown in Table 1.(7) Since VISSIM is a behavior-based program, the adjusted and valid ranges of the parameters for calibration were set by analyzing the flow-speed data collected from the Gyeongbu Expressway in order to represent accurate traffic conditions in the model. The valid ranges were set within the adjusted ranges. 
Table 1. Car Following Model Parameters(7)

\begin{tabular}{cccc}
\hline Parameter & Description & Default value & Unit \\
\hline \hline CC0 & Standstill Distance & 1.50 & $\mathrm{~m}$ \\
CC1 & Headway time & 0.90 & $\mathrm{~s}$ \\
CC2 & Following Variation & 4.00 & $\mathrm{~m}$ \\
CC3 & Threshold for Entering Following & -8.00 & \\
CC4 & Negative Following Threshold & -1.50 & \\
CC5 & Positive Following Threshold & 1.50 & \\
CC6 & Speed dependency of Oscillation & 11.44 & \\
CC7 & Oscillation Acceleration & 0.25 & $\mathrm{~m} / \mathrm{s}^{2}$ \\
CC8 & Standstill Acceleration & 3.50 & $\mathrm{~m} / \mathrm{s}^{2}$ \\
CC9 & Acceleration at 80 km/h & 1.50 & $\mathrm{~m} / \mathrm{s}^{2}$ \\
\hline
\end{tabular}

The parameters of the driver behavior model, whose adjusted and valid ranges were calculated from the flow-speed data, are demonstrated in Table 2.

Table 2. Driver Behavior Model Parameters

\begin{tabular}{cccc}
\hline Parameter & Default Value & Adjusted Ranges & Valid Ranges \\
\hline \hline CC0 & 1.50 & $0.5 \sim 4.0$ & $0.5 \sim 1.0$ \\
CC1 & 0.90 & $0.5 \sim 3.0$ & $0.5 \sim 1.5$ \\
CC2 & 4.00 & $2.0 \sim 20.0$ & $2.0 \sim 4.0$ \\
CC4/5 & 0.35 & $0.1 \sim 2.0$ & $0.5 \sim 1.0$ \\
CC7 & 0.25 & $0.1 \sim 1.0$ & $0.1 \sim 1.0$ \\
\hline
\end{tabular}

Standstill Distance(CC0), also known as the clear space, defines the desired distance between stopped cars. As this parameter affects drivers when they keep their safety distance, it affects the road capacity. The default value was set at $1.5 \mathrm{~m}$. The adjusted range was set to $0.5 \sim 4.0 \mathrm{~m}$ based on the analysis of the flow-speed data collected from the Gyeongbu Expressway and after further analysis with the adjusted range, the valid range was set at $0.5 \sim 1.0 \mathrm{~m}$, as shown in Figure 6 . Headway Time $(\mathrm{CC} 1)$ defines the minimum time required to maintain a safe distance between drivers. Like $\mathrm{CC} 0$, this parameter affects road capacity and its default value was set at 0.90 seconds. The adjusted range was set to $0.5 \sim 4.0$ seconds and after further analysis with this range, the valid range was set to $0.5 \sim 1.5$ seconds, as shown in Figure 7.

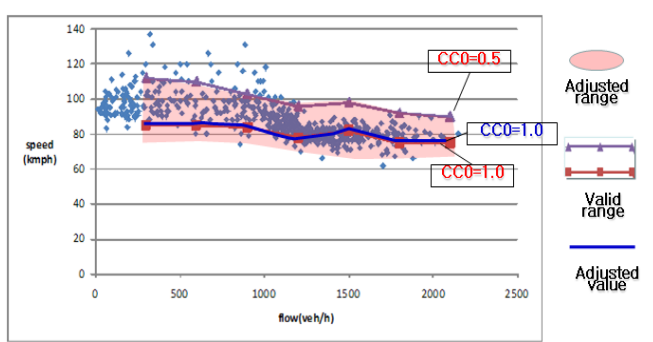

Figure 6. Parameter CCO Adjustment

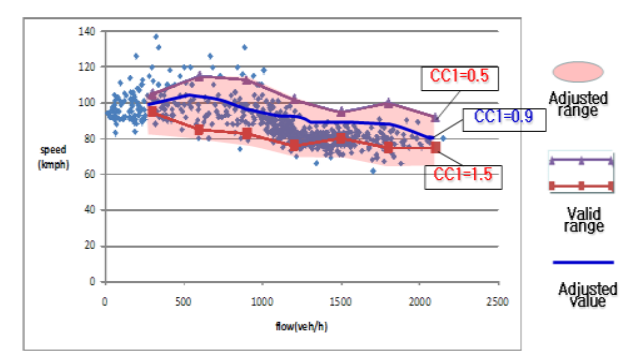

Figure 7. Parameter CC1 Adjustment

Following Variation(CC2) is an element that limits the following variation in a simulation. The following variation is a distance larger than the safety distance between 
drivers. The default value was set at $4.00 \mathrm{~m}$. The adjusted range of CC2 was analyzed as 2.0 20.0 and after further analysis with the adjusted range, the valid range was set to 2.0 4.0, as shown in Figure 8. Following Thresholds(CC4 and CC5) are parameters that control the variation of acceleration and deceleration of the vehicle in front. The driver's reaction to the acceleration and deceleration of the car in front is more sensitive when this value is lower. The default value was set at \pm 0.35 . The adjusted range was analyzed as 1.0 2.0 and after further analysis with the adjusted range, the valid range was set to $0.5 \sim 1.0$, as shown in Figure 9.

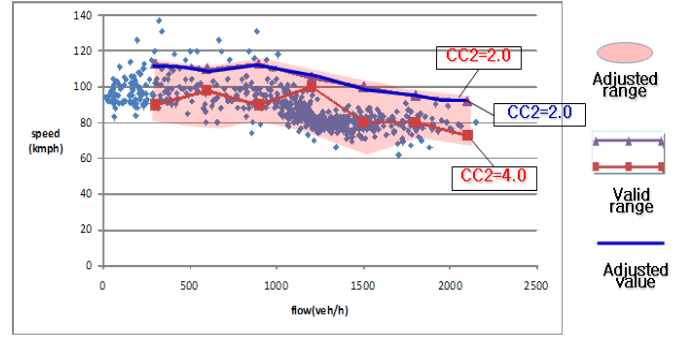

Figure 8. Parameter CC2 Adjustment

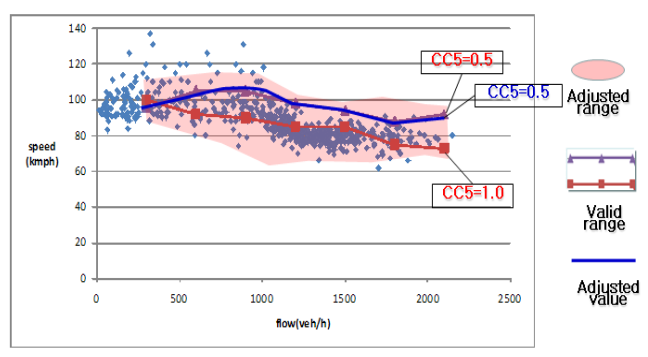

Figure 9. Parameters CC4 and CC5 Adjustment

The valid ranges for each parameter, shown in Figures 6, 7, 8 and 9, are applied on the model to analyze the Gyeongbu Expressway. The parameter values that optimized the simulation model were determined and defined as the adjusted values shown in Table 3.

Table 3. Driver Behavior Model Application

\begin{tabular}{llcl}
\hline Parameter & Description & Valid Ranges & Adjusted Value \\
\hline \hline CC0 & Standstill Distance & $0.5 \sim 1.0$ & 1.0 \\
CC1 & Headway time & $0.5 \sim 1.5$ & 0.9 \\
CC2 & Following Variation & $2.0 \sim 4.0$ & 2.0 \\
CC4/5 & Following Threshold & $0.5 \sim 1.0$ & 0.5 \\
CC7 & Oscillation Acceleration & $0.1 \sim 1.0$ & 0.25 \\
\hline
\end{tabular}

GEH Index(8) (ED what's 'GEH Index'?) of this model with the adjusted values in Table 3 was 3.87, which was an accepted value.

$$
G E H=\sqrt{\frac{2(\mathrm{Obs} \mathrm{Val}-\operatorname{Sim} \mathrm{Val})^{2}}{O b_{s} \mathrm{Val}+\operatorname{Sim} \mathrm{Val}}}
$$

Satisfactorily calibrated if:

GEH<5.00: for $85 \%$ of the links and

$\mathrm{GEH}<4.00$ for the sum of all link counts

Where,

ObsVal: Observed volume (number of vehicles/hour)

SimVal: Simulated volume (number of vehicles/hour)

In comparison of the real data collected from the Gyeongbu Expressway and the output data of the model with the adjusted values, there was no significant difference in terms of frequency and travel speed, as shown in Table 4 and Figure 10. 
Table 4. Number of Links and GEH Index

\begin{tabular}{llll}
\hline No. of Links & Observed Volume & Simulated Volume & GEH Value \\
\hline \hline Northbound & 6,439 & 6,227 & 2.66 \\
Southbound & 5,322 & 5,124 & 3.02 \\
Total & 11,761 & 11,351 & 3.87 \\
\hline
\end{tabular}

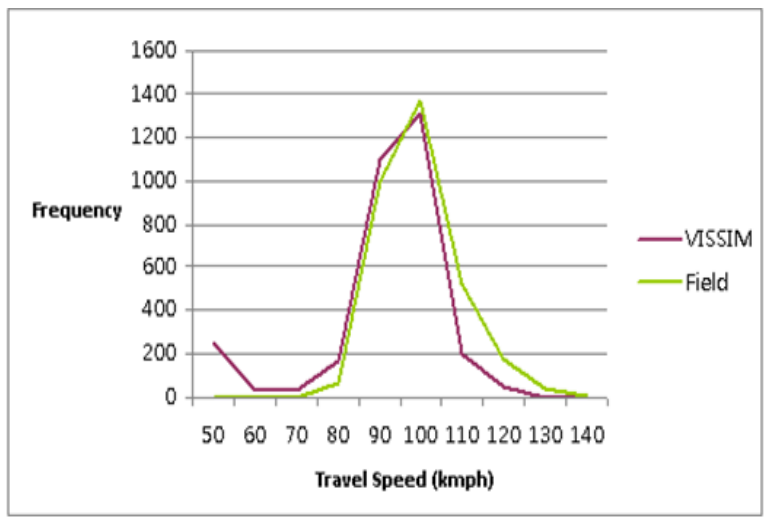

Figure 10. Speed Frequency Comparison between Observed and Modeled Data

\section{Simulation Model Application}

\subsection{Application to HOV Lane}

Based on the previous parameters of the driver behavior model for the Gyeongbu Expressway, parameters for HOV lanes were also adjusted through a feedback process, as shown in Table 5. The HOV lane parameters had lower values than the general ones, indicating that the drivers using the HOV lane were more aggressive than the others, because most of the vehicles in the HOV lane were buses with more experienced drivers than drivers on general lanes in that area. The travel speed variations due to HOV lane enforcement during the morning peak hours were analyzed by VISSIM program. After the enforcement, the average travel speed of the HOV vehicles was increased by about $30 \mathrm{~km} / \mathrm{h}$ from $38 \mathrm{~km} / \mathrm{h}$ to $68 \mathrm{~km} / \mathrm{h}$, thereby reducing their travel time by about 30 to $40 \mathrm{mins}$, while that of the other cars was decreased by $10 \mathrm{~km} / \mathrm{h}$ from $45 \mathrm{~km} / \mathrm{h}$ to $35 \mathrm{~km} / \mathrm{h}$, thereby increasing their travel time by about 15 to $20 \mathrm{mins}$, compared to the values before the enforcement (Table 6). This should encourage car users to use public transport.

Table 5. Adjusted Parameters for HOV Lane

\begin{tabular}{llll}
\hline Parameter & Description & General Lane & HOV Lane \\
\hline \hline CC0 & Standstill Distance & 1.0 & 0.50 \\
CC1 & Headway time & 0.9 & 0.70 \\
CC2 & Following Variation & 2.0 & 2.0 \\
CC4/5 & Following Threshold & 0.5 & 0.50 \\
CC7 & Oscillation Acceleration & 0.25 & 0.25 \\
\hline
\end{tabular}


Table 6. Travel Speed before/after HOV Lane Enforcement (AM Peak Hours)

\begin{tabular}{llll}
\hline HOV & & Car & \\
\hline \hline Before & After & Before & After \\
$38 \mathrm{~km} / \mathrm{h}$ & $68 \mathrm{~km} / \mathrm{h}$ & $45 \mathrm{~km} / \mathrm{h}$ & $35 \mathrm{~km} / \mathrm{h}$ \\
\hline
\end{tabular}

\subsection{Application to Movable Lane}

With movable lane enforcement, as an example presented in Figure 11, the number of lanes can be managed according to the traffic volumes in each direction. There are 8 lanes in both directions in the study area, from Seoul Toll Gate to Osan Interchange. In this paper, the numbers of lanes towards Seoul and towards Osan were set to 5 and 3, respectively, due to the heavier traffic towards Seoul during the morning peak hours.

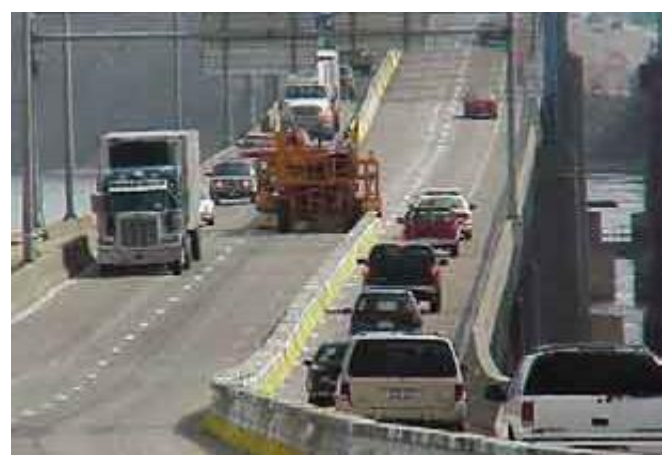

Figure 11. Movable Lane ${ }^{1}$

The parameters for the movable lanes were adjusted through a feedback process of driver behavior-based model for the Gyeongbu Expressway. The parameters representing driver attribute were set to a value between the general lanes and the HOV lanes, as shown in Table 7.

Table 7. Adjusted Parameters for Movable Lane

\begin{tabular}{lllll}
\hline Parameter & Description & General Lane & HOV Lance & Movable Lane \\
\hline CC0 & Standstill Distance & 1.00 & 0.50 & 1.00 \\
CC1 & Headway time & 0.90 & 0.70 & 0.80 \\
CC2 & Following Variation & 2.00 & 2.00 & 2.00 \\
CC4/5 & Following Threshold & 0.50 & 0.50 & 0.50 \\
CC7 & Oscillation Acceleration & 0.25 & 0.25 & 0.25 \\
\hline
\end{tabular}

Travel speed variations due to movable lane enforcement during morning peak hours were also analyzed by the VISSIM program. The average travel speed of vehicles towards Seoul was increased by about $9 \mathrm{~km} / \mathrm{h}$ from $45 \mathrm{~km} / \mathrm{h}$ to $54 \mathrm{~km} / \mathrm{h}$, while that in the other direction was decreased by about $7 \mathrm{~km} / \mathrm{h}$ from $68 \mathrm{~km} / \mathrm{h}$ to $61 \mathrm{~km} / \mathrm{h}$ due to the reduction in lanes.

1 Source : www.tfhrc.gov/pubrds/04nov/04.htm 
In terms of the total system performance, the total travel cost was decreased due to the greater increase in the average travel speed in the Seoul direction than the decrease in the other direction, and the greater traffic flow in the former than the latter, as shown in Table 8.

Table 8. Travel Speed before/after Movable Lane Enforcement (AM Peak Hours)

\begin{tabular}{cccc}
\hline \multicolumn{2}{c}{ Towards Seoul } & \multicolumn{2}{c}{ Towards Osan } \\
\hline \hline Before & After & Before & After \\
$45 \mathrm{~km} / \mathrm{h}$ & $54 \mathrm{~km} / \mathrm{h}$ & $68 \mathrm{~km} / \mathrm{h}$ & $61 \mathrm{~km} / \mathrm{h}$ \\
\hline
\end{tabular}

\subsection{Effect Comparison}

With HOV lane enforcement, the travel speed of the HOV vehicles significantly increased from $32 \mathrm{~km} / \mathrm{h}$ to $68 \mathrm{~km} / \mathrm{h}$ while that of the total vehicles dropped from $45 \mathrm{~km} / \mathrm{h}$ to $37 \mathrm{~km} / \mathrm{h}$.

With movable lane enforcement, the travel speed of not only the HOV vehicles but also the total vehicles increased to $48 \mathrm{~km} / \mathrm{h}$ and $54 \mathrm{~km} / \mathrm{h}$, respectively, as shown in Table 9.

Table 9. Comparison of Travel Speed

\begin{tabular}{cccc}
\hline Travel Speed & Now & HOV Lane Enforcement & Movable Lane Enforcement \\
\hline \hline HOV Vehicles & $32 \mathrm{~km} / \mathrm{h}$ & $68 \mathrm{~km} / \mathrm{h}$ & $48 \mathrm{~km} / \mathrm{h}$ \\
Total Vehicles & $45 \mathrm{~km} / \mathrm{h}$ & $37 \mathrm{~km} / \mathrm{h}$ & $54 \mathrm{~km} / \mathrm{h}$ \\
\hline
\end{tabular}

\section{Conclusion}

Two sustainable transportation alternatives were analyzed in this study. Firstly, in order to encourage people to use public transportation, HOV lane enforcement was evaluated. Secondly, in order to improve car efficiency, managed lane schemes with movable median lane enforcement were evaluated. VISSIM, a microscopic, behaviorbased, traffic simulation program, was used for this study and the parameters representing driver behavior in the car following model were adjusted through the calibration process by using the measured data in the Gyeongbu Expressway.

The analysis results showed that the bus travel speed increased markedly with the HOV lane enforcement, thus saving passengers' travel time. In addition, the movable median lane enforcement improved car efficiency due to the increased travel speed of the total vehicles.

In conclusion, the managed lane schemes improved the level of service of public transportation, which holds the promise of shifting car users to public transportation and contributing to a sustainable transportation system by reducing the energy consumed by land vehicles with improved car efficiency.

\section{Acknowledgments}

This work was supported by the National Research Foundation of Korea Grant funded by the Korean Government (No. NRF-2015R1A2A2A04005646) 


\section{References}

[1] D. Yoon and Y. Kim, "The Study of Performance Measures Index Development for Sustainable Transportation Planning”, Journal of Transportation Research Society of Korea, (2000), pp. 135-148.

[2] J. Park, "A trend of Sustainable Transportation Policy and Implication of the Auto Industry", Reports of the Korea Transportation Institute, (2008).

[3] H. Lee and Y. Lee, "Vehicle travel distance Research", Reports of the Korea Transportation Safety Authority, (2007).

[4] F. Choa, R. Millam and D. Stanek, "CORSIM, PARAMICS, and VISSIM: What the Manuals Never Told You" ITE Conference. (2002).

[5] S. Chundry and B. Wolshon, "Evaluation of the CORSIM Car-Following Model Using GPS Field Data", 79th Annual Meeting of the Transportation Research Board. CD-ROM. Washington, D.C., (2000).

[6] M. Fellendorf and P. Vortisch, "Validation of the Microscopic Traffic Flow Model VISSIM in Different Real-World Situations", Proceedings of the 80th Annual Meeting of the Transportation Research Board, Washington, DC., (2001).

[7] E. Nicholas and B. Randy, "A multi-parameter sensitivity analysis", Proceedings of the 2006 Winter Simulation Conference, (2004).

[8] Richard, D., Alexander, S., and John, H.: "Guidelines for Calibration of Microsimulation Models", Journal of the Transportation Research Board, (2004) 